\title{
Comparative analysis of fungal protein kinases and associated domains
}

\author{
Idit Kosti ${ }^{1}$, Yael Mandel-Gutfreund ${ }^{1}$, Fabian Glaser ${ }^{2 *}$, Benjamin A Horwitz ${ }^{1 *}$
}

\begin{abstract}
Background: Protein phosphorylation is responsible for a large portion of the regulatory functions of eukaryotic cells. Although the list of sequenced genomes of filamentous fungi has grown rapidly, the kinomes of recently sequenced species have not yet been studied in detail. The objective of this study is to apply a comparative analysis of the kinase distribution in different fungal phyla, and to explore its relevance to understanding the evolution of fungi and their taxonomic classification. We have analyzed in detail 12 subgroups of kinases and their distribution over 30 species, as well as their potential use as a classifier for members of the fungal kingdom.

Results: Our findings show that despite the similarity of the kinase distribution in all fungi, their domain distributions and kinome density can potentially be used to classify them and give insight into their evolutionary origin. In general, we found that the overall representation of kinase groups is similar across fungal genomes, the only exception being a large number of tyrosine kinase-like (TKL) kinases predicted in Laccaria bicolor. This unexpected finding underscores the need to continue to sequence fungal genomes, since many species or lineage-specific properties may remain to be discovered. Furthermore, we found that the domain organization significantly varies between the fungal species. Our results suggest that protein kinases and their functional domains strongly reflect fungal taxonomy.
\end{abstract}

Conclusions: Comparison of the predicted kinomes of sequenced fungi suggests essential signaling functions common to all species, but also specific adaptations of the signal transduction networks to particular species.

\section{Background}

Filamentous fungi detect and respond to a variety of signals. As saprophytes or parasites, fungi need to monitor the nutrient status, presence of a host and host defenses, and avoid or respond to osmotic or oxidative stress, light and other environmental variables [1]. Although fungal development is less complex than that of higher multicellular eukaryotes, internal signals are probably required to program major differentiation steps: hyphal extension and branching, sporulation, mating, secondary metabolite accumulation and production of infection structures. Finally, regulation of transcription, translation and cell division is expected to be similar to that of other eukaryotes. Indeed, fungal models such as budding

\footnotetext{
*Correspondence: fglaser@tx.technion.ac.il; horwitz@tx.technion.ac.il ${ }^{1}$ Department of Biology, Technion - Israel Institute of Technology, Haifa ${ }^{1}$ Department of Biology, Technion - Israel Institute of Technology, Haifa
32000, Israel

${ }^{2}$ Bioinformatics Knowledge Unit, The Lorry I. Lokey Interdisciplinary Center for Life Sciences and Engineering, Technion - Israel Institute of Technology, Haifa 32000, Israel
}

(c) 2010 Kosti et al; licensee BioMed Central Ltd. This is an Open Access article distributed under the terms of the Creative Commons Attribution License (http://creativecommons.org/licenses/by/2.0), which permits unrestricted use, distribution, and reproduction in any medium, provided the original work is properly cited. and fission yeasts, Neurospora and Aspergillus have figured prominently in cell biology and genetics [2].

Protein kinases have roles in every aspect of regulation and signal transduction, and provide new targets for drug development [3]. Most eukaryotic protein kinases, with the exception of the histidine kinases found in two-component sensory systems, belong to a superfamily defined by a conserved protein kinase domain (ePK, eukaryotic protein kinase). There are nearly 500 members of this superfamily in human and mouse [4-6]. The ePK superfamily can be classified into several major groups. The members of each of these groups are related according to the signals that activate them and the kinds of protein substrates they phosphorylate. The original classification of Hanks and Hunter [7] has been extended, refined, and applied to many eukaryotic genomes including some fungi [8]. Beginning a few years ago there has been an increasing effort to sequence filamentous fungal genomes [9]. There has, however, been no complete study of the kinomes of the recently 
sequenced filamentous fungi. Furthermore, in addition to identifying and classifying the kinases in the genomes, there is now a unique opportunity to discover speciesspecific properties, as well as general trends related to taxonomic group or other properties that are shared between different sequenced fungi.

Based on the kinomer database [8], we developed an automatic pipeline that predicts all putative kinases from any given proteome, and classifies them. We then analyzed their distribution, and tested different potential classification methods. In addition to the basic ePK domain present in almost all putative kinases, other domains of the protein are essential for kinase activity and interaction with effector proteins and substrates. In the human kinome, 83 additional domain types were identified, and additional domains were recognized in over half of the kinases [6]. We identified all additional domains that are present in the fungal kinomes according to PFAM classification, and discuss their presence or absence in the different groups. Finally, we show that the information contained in the domains is sufficient to classify the fungi. Our analysis can be applied to any other taxonomic or protein groups, and suggests a great functional richness of kinases in different organisms.

\section{Results and Discussion}

We have studied the distribution, domain content and kinase density among 30 species representing the Dikarya or higher fungi, including two phyla (see Table 1). In this analysis, we obtained the full proteome for each fungal genome from diverse sources, and then used the Kinomer database [10] to identify and classify each predicted kinase. This database classifies the eukaryotic protein kinases into two groups: 'conventional' (ePK) and 'atypical' (aPKs) protein kinases. This classification, based on sequence similarity, also allows the construction of an accurate multi-level HMM library that can be used to search and classify each putative kinase in any organism to each of the 12 basic subgroups (see Methods for details).

\section{Distribution of kinases in each functional sub-group}

The initial result of this work is a list of putative kinase proteins, classified by the Kinomer library. Figure 1A shows the distribution of the 11 populated groups of kinases (RGC has no representatives) found by applying the Kinomer HMM library to each of the 30 fungal proteomes studied. The main populated groups of kinases are AGC, CMAK, CMGC and STE, all of them belonging to the protein kinase superfamily $[7,10]$. These four groups include $88 \%$ of all predicted kinases on average. The least populated groups are Alpha and TK with only 1 and 2 representatives for all fungi, respectively. Within the Hemiascomycota group (ascomycete yeasts: subphylum Saccharomycotina) the number and distribution of the different kinase groups are generally similar (Figure 1A). In contrast, within the filamentous Ascomycota (subphylum Pezizomycotina) and the Basidiomycota groups, the variation in terms of kinase number is much higher. When we look, however, at the normalized frequency of kinases in each group (relative to the total number of kinases in each fungal proteome, Figure $1 \mathrm{~B})$, it is evident that, despite minor variations within the different phyla and subphyla, the overall proportion of each group is quite similar in each kinase sub-group: AGC includes about $20 \%$ of all kinases in each species, CAMK about $30 \%$, etc.

Thus, most variation between species diminishes upon normalization. Nevertheless, in terms of the kinome size, we can see two distinct groups. The first one has larger kinomes with, on average, 159 kinases, and includes the ascomycetes Aspergillus clavatus, Aspergillus niger, Neosartorya fischeri, Aspergillus nidulans and Ascosphaera apis and the basidiomycetes Cryptococcus neoformans and Malassezia globosa. The second group includes all other fungi in our study, having an average of 85 putative kinases (see Additional File 1). Thus, the larger group has, on average, almost twice the number of kinases compared to the smaller group. The large variation in the total number of kinases might be related to partial genome duplication event(s). This assumption is supported by considerable evidence of genome duplication in fungi [11]. However, expansion of the number of predicted protein kinases does not correspond to the overall predicted frequency of duplications. Aspergillus nidulans, for example, has an expanded number of kinases compared to A. fumigatus and A. oryzae (as we find also in this study, see Additional File 1), yet the estimated frequency of duplications [11] is quite similar for these three members of the genus Aspergillus. About a third of our samples seem to have undergone a possible duplication event of either the kinome and/or the genome of these fungi.

As mentioned above, the Hemiascomycota (Ascomycota, Subphylum Saccharomycotina) group seems to be much more compact in terms of kinase number. This indicates, perhaps, a "tighter" evolution of this group with respect to their signaling pathways. For those fungi that have not undergone kinome duplication, the number of kinases found for each group is remarkably similar (see black bars in Additional File 1), given the large evolutionary differences between them. The reasons for independent expansion of protein kinase gene families are not clear, but it has been proposed that genes involved in regulatory interactions might retain duplication. The result would be selective expansion of these classes of genes. Modeling of the effects of three genome duplications in Arabidopsis over the past 350 
Table 1 Species included in the kinome analyses.

\begin{tabular}{|c|c|c|c|c|c|}
\hline & Fungal species & Abbreviation & Phylum/Subphylum/Class* & Database & Genome Paper \\
\hline 1 & Ascosphaera apis & Aapis_as & Ascomycota/P/E & Baylor/NCBI & {$[31]$} \\
\hline 2 & Aspergillus clavatus & Aclavatus_as & Ascomycota/P/E & NCBI Protein & {$[32]$} \\
\hline 3 & Aspergillus fumigatus & Afumigatus_as & Ascomycota/P/E & Broad Institute & {$[28,33]$} \\
\hline 4 & Aspergillus nidulans & Anidulans_as & Ascomycota/P/E & Broad Institute & {$[28,33]$} \\
\hline 5 & Aspergillus niger & Aniger_as & Ascomycota/P/E & NCBI Protein & {$[34]$} \\
\hline 6 & Aspergillus oryzae & Aoryzae_as & Ascomycota/P/E & Broad Institute & {$[28,33]$} \\
\hline 7 & Coccidioides immitis & Cimmitis_as & Ascomycota/P/E & Broad Institute & {$[35]$} \\
\hline 8 & Fusarium graminearum & Fgraminearum_as & Ascomycota/P/So & Broad Institute & {$[36]$} \\
\hline 9 & Histoplasma capsulatum. & Hcapsulatum_as & Ascomycota/P/E & Broad Institute & {$[35]$} \\
\hline 10 & Magnaporthe oryzae (grisea) & Mgrisea_as & Ascomycota/P/So & Broad Institute & {$[37]$} \\
\hline 11 & Neurospora crassa & Ncrassa_as & Ascomycota/P/So & Broad Institute & {$[38]$} \\
\hline 12 & Neosartorya fischeri & Nfischeri_as & Ascomycota/P/E & Venter & {$[32]$} \\
\hline 13 & Penicillium chrysogenum & Pchrysogenum_as & Ascomycota/P/E & NCBI Protein & [39] \\
\hline 14 & Stagonospora nodorum & Snodorum_as & Ascomycota/P/D & $J G l$ & {$[40]$} \\
\hline 15 & Sclerotinia sclerotiorum & Ssclerotiorum_as & Ascomycota/P/L & Broad Institute & In preparation \\
\hline 16 & Trichoderma reesei & Treesei_as & Ascomycota/P/So & $J G l$ & [41] \\
\hline 17 & Uncinocarpus reesii & Ureesii_as & Ascomycota/P/E & Broad Institute & [35] \\
\hline 18 & Cryptococcus neoformans & Cneoformans_ba & Basidiomycota/A & Broad Institute & {$[42]$} \\
\hline 19 & Laccaria bicolor & Lbicolor_ba & Basidiomycota/A & $\mathrm{JGl}$ & {$[43]$} \\
\hline 20 & Malassezia globosa & Mglobosa_ba & Basidiomycota/U & NCBI Protein & {$[44]$} \\
\hline 21 & Phanerochaete chrysosporium & Pchrysosporium_ba & Basidiomycota/A & $J G l$ & {$[45]$} \\
\hline 22 & Ustilago maydis & Umaydis_ba & Basidiomycota/U & Broad Institute & [46] \\
\hline 23 & Ashbya (Eremothecium) gossypii & Agossypii_he & Ascomycota/S & NCBI Protein & {$[47]$} \\
\hline 24 & Candida albicans & Calbicans_he & Ascomycota/S & Broad Institute & {$[48]$} \\
\hline 25 & Candida glabrata & Cglabrata_he & Ascomycota/S & Genolevures & [49] \\
\hline 26 & Debaromyces hansenii & Dhansenii_he & Ascomycota/S & Genolevures & [49] \\
\hline 27 & Kluyveromyces lactis & Klactis_he & Ascomycota/S & Genolevures & {$[49]$} \\
\hline 28 & Pichia stipitis & Pstipitis_he & Ascomycota/S & $\mathrm{JGl}$ & {$[50]$} \\
\hline 29 & Saccharomyces cerevisiae & Scereviseae_he & Ascomycota/S & SGD & {$[51]$} \\
\hline 30 & Schizosaccharomyces pombe & Spombe_as & Ascomycota/T & Sanger & {$[2]$} \\
\hline
\end{tabular}

Taxonomy follows [18] and [21]. Saccharomycotina (Ascomycete yeasts) are often referred to as Hemiascomycota and we have retained the tag _he in the abbreviated species names in the figures, for simplicity.

*Subphylum: U, Ustilaginomycotina; A, Agaricomycotina; P, Pezizomycotina; S, Saccharomycotina; T, Taphrinomycotina; Class: So, Sordariomycetes; L, Leotiomycetes, E, Eurotiomycetes; D, Dothideomycetes.

million years suggests that duplications of regulatory genes are retained, particularly, for large-scale events, because signal transducer proteins act in complexes [12,13]. The Zygomycete Rhizopus oryzae genome has undergone a high level of duplication [14]. In this species, we found (data not shown) that the main kinase families are expanded about two fold. Here again, after normalization the relative number of kinase families are within the range of the Dikarya which are shown in Figure 1B. Analysis of other Zygomycete species will answer the question of whether expanded numbers of kinases are a more general property of this phylum.

One striking exception to the overall similarity in the (normalized) distribution of kinases among the major subfamilies is the TKL kinase family (25) predicted for Laccaria bicolor. It is generally thought that there are no tyrosine kinases (TK) in fungi and our results support this. Nevertheless, in our data the Basidiomycete Laccaria bicolor shows, exceptionally, a huge number of TKL protein kinase genes. Pending direct experimental evidence, of course, this suggests a large deviation of this fungus kinome towards the TKL group.

\section{Kinome and Proteome Size}

An expanded and/or diverse kinome may provide a more flexible signaling network. This implies that overall parameters like kinome size as compared to proteome size might not always follow classical (and 


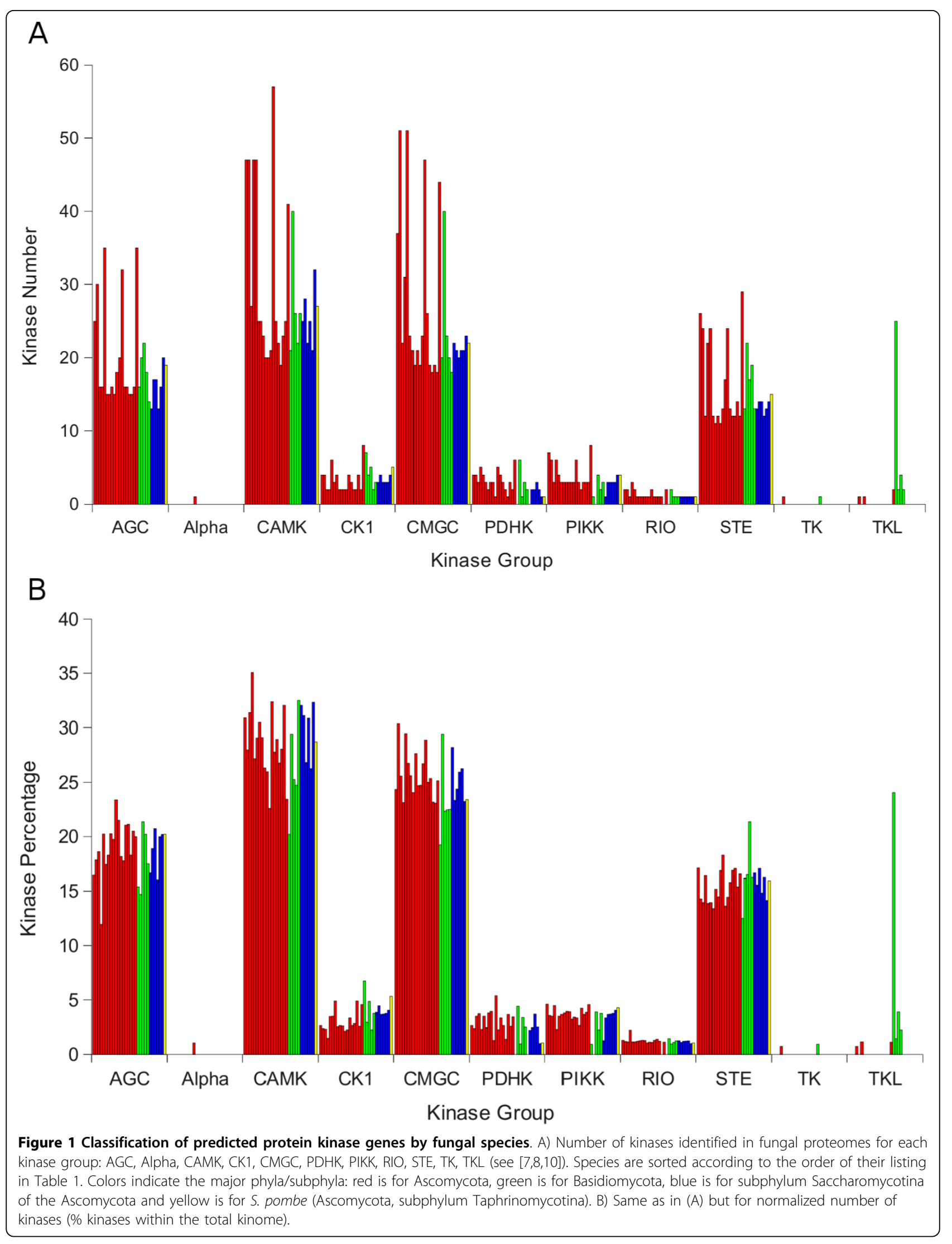


molecular) taxonomy. A striking example comes from recent work on the genomes of myxobacteria, which are prokaryotic, but contain an unexpectedly high number of eukaryotic-like (serine/threonine and tyrosine) protein kinases [15]. The proteome and kinome sizes of the fungi used in this study vary considerably. We note that the predicted fungal proteomes are only as accurate as the assumptions used in their construction. For example alternative splicing, a major source of protein diversity, is not taken into account, although there is evidence for biologically important alternative splicing events in fungi (in the Neurospora circadian clock, for example, $[16,17])$. Figure $2 \mathrm{~A}$ shows the correlation between proteome and kinome sizes. The Hemiascomycota (Ascomycota, Saccharomycotina) fungi form a fairly uniform cluster (blue squares on cluster 1, Figure 2B). These species have smaller proteomes and tend to be highly similar in terms of kinome and proteome size. The Ascomycota (Pezizomycotina) and Basidiomycota groups have larger proteomes and also show greater variation; a subset of the filamentous Ascomycetes forms a compact group, quite variable in proteome size, but with very similar kinome sizes (cluster 2, Figure 2A).

Another way to look at this information would be to calculate the kinome density. This is the percentage of the total number of predicted kinase proteins within the total number of proteins predicted for each fungal species. Figure $2 \mathrm{~B}$ shows the kinase density for all the fungi, sorted by value. It suggests that filamentous ascomycetes and the hemiascomycete yeasts can be classified according to their kinome density. The basidiomycetes have a much more variable kinome density and therefore it would be difficult to classify them based on this parameter. A Wilcoxon test ( $\mathrm{p}$-value = $9.6 \times 10^{-4}$ ) confirmed that the Ascomycete and Hemiascomycete fungi could be successfully classified using their kinome density values. This difference in kinome density might represent the result of evolutionary pressure toward diversification of signal transduction pathways. Although this seems logical, there is really no obvious correlation between kinome density or diversity and the "lifestyle" (pathogen or saprophyte, particular host or ecological niche) of the sequenced species that we have studied. S. pombe is the only exception within the Ascomycetes, having the largest kinome density of all fungi studied. S. pombe though is the only species belonging to the Taphrinomycotina subphylum within the Ascomycetes, which represents its own subphylum branch. Thus we cannot anticipate whether this is a unique difference or a trend of its subphylum (see Figure 1 in [18]). Interestingly, the second densest fungal kinome belongs to S. cereviseae, another type of yeast.

\section{Domain distribution}

Most protein kinases act in combination with other kinases and other signaling effectors, and are modulated by phosphorylation cascades. Other domains within these proteins have important regulatory activity, link to other signaling modules, or provide a localization signal [6]. We therefore studied the identity and number of domains flanking the kinase catalytic domain ePK in each predicted kinase. To this aim we searched the putative kinases against the PFAM database (see Methods).

The 30 fungal species have a total of 2976 putative kinase sequences, matching 4294 significant PFAM domains, which makes an average of 1.4 domains per kinase. According to PFAM, 3292 domains have kinase catalytic activity and the remaining 1002 have nonkinase domain activity. This suggests that the kinase proteins have an enormous richness of functional domains, with an average 0.3 of non-kinase domains and 1.1 kinase domains per sequence. We find that there are a total of 72 different domain types, of which 7 are annotated in PFAM to have kinase activity (see Figure 3 and text below): Pkinase (2867 domains), Pkinase_C (196), PI3_PI4_kinase (103), BCDHK_Adom3 (81), RIO1 (36), Pkinase_Tyr (8) and Alpha_kinase (1). The Pkinase domain is the most common type of kinase domain in our fungi, where it represents about $87 \%$ of all catalytic domains, and in PFAM, with more than 32000 representative sequences. But Pkinase is not the only conserved kinase catalytic domain type. There are 425 additional putative kinases with a catalytic kinase domain different from the classical kinase catalytic domain Pkinase: Pkinase_C is a kinase $C$ terminal domain, PI3_PI4_kinase is a phosphatidylinositol 3- and 4-kinase domain, BCDHK_Adom3 is a mitochondrial dehydrogenase kinase domain, RIO1 is a typical serine kinase domain, the Pkinase_Tyr kinase, a tyrosine kinase domain and Alpha_kinase an alpha kinase domain. Those additional kinase domains represent almost 13\% of the catalytic kinase domains, and add a rich variety of specific kinase catalytic functions to the kinome.

Very interestingly, while Pkinase (and in smaller proportion Pkinase_C, PI3_PI4_kinase, BCDHK_Adom3, HATPase_c and RIO1) appears almost in every fungal species, Pkinase_Tyr and Alpha_kinase domains are much more rare (see below). Pkinase_Tyr is highly represented only in Laccaria bicolor while isolated TK and TKL kinases were found in several species in our analysis and in the Kinomer database [10].

Figure 3 shows the sum of the domain distribution found in the predicted kinase proteins studied here. Overall, we have found that in addition to the 7 kinase domains, there are 65 different types of domains. This number is about three quarters of that found for the 

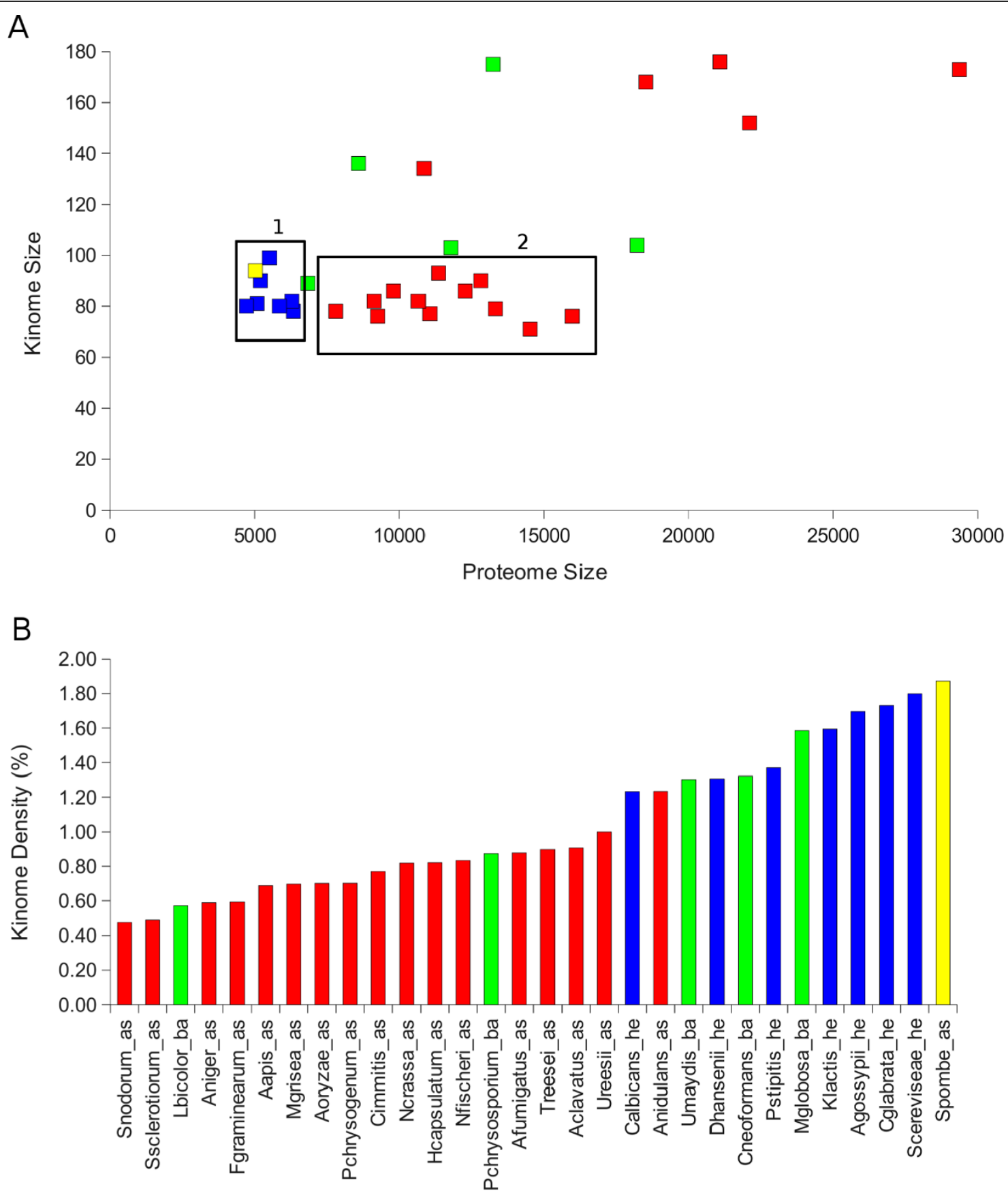

Figure 2 Correlation between kinome size and proteome size. Colors are as in Figure 1. A) Scatter plot: two clusters that can be inferred from this plot are indicated. B) Kinome density values (total number of kinases/proteome size).

human kinome [6]. The three most common domains are kinase catalytic domains (in black in Figure 3), while many of the highly frequent non-kinase domains (in grey in Figure 3) are kinase regulatory domains, like FHA, FATC, HR1, etc. Additionally, Figure 3 shows that there are 32 very rare domains, appearing only once in all the kinomes studied, like TPP_enzyme_C (Thiamine pyrophosphate enzyme, $\mathrm{C}$-terminal TPP binding domain) and
Fungal_trans domain (Fungal specific transcription factor domain). This variation in the domain type and frequency indicates functional and evolutionary differences that are not easy to interpret, but that can, in principle, be used to classify the fungi, as a complement to classical taxonomical and phylogenetic procedures.

Of the first 10 most common domains in the fungi studied here, 9 are also present in the human kinome, 


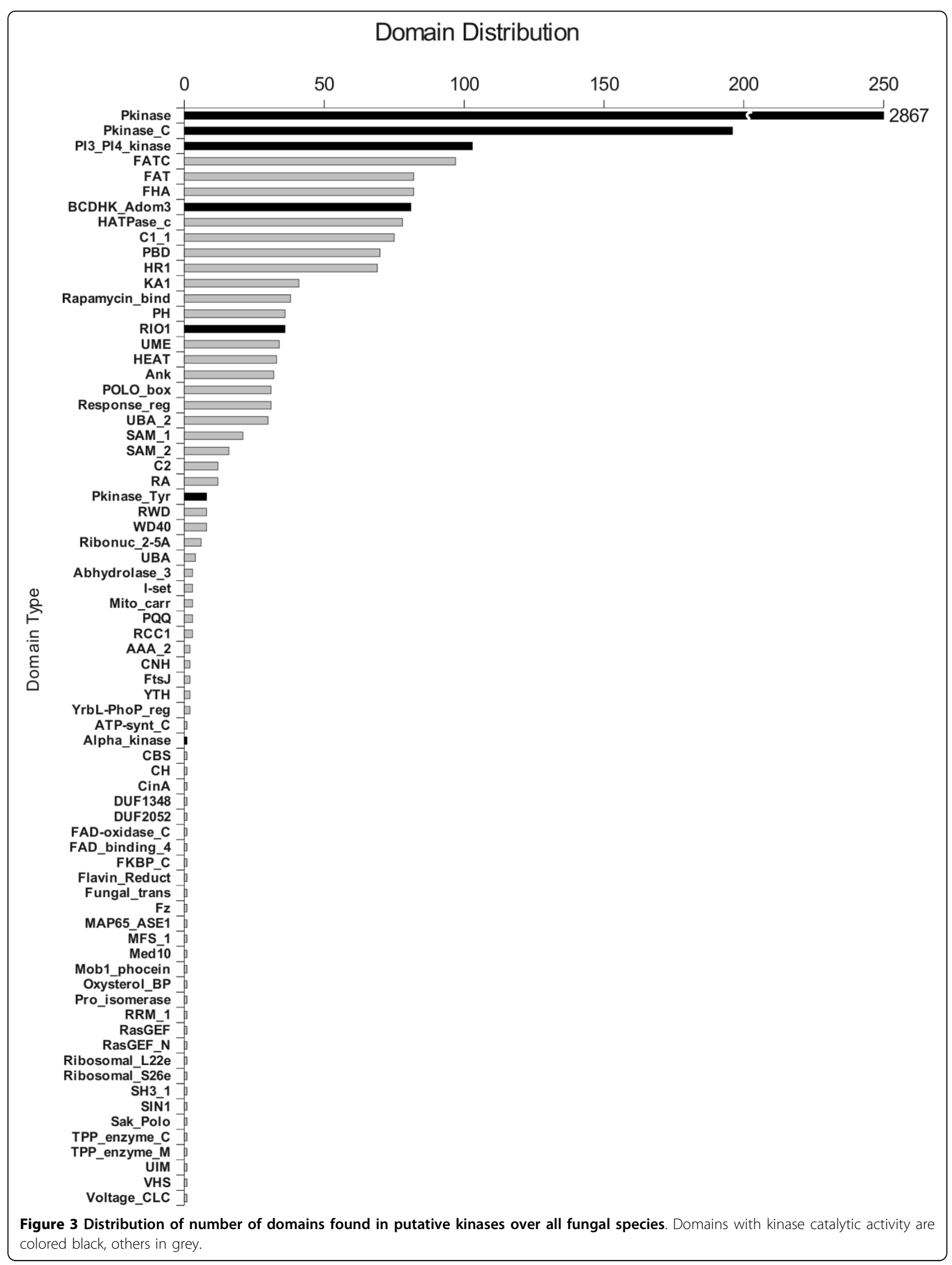


reflecting a highly conserved kinome functional milieu: Pkinase_C (Catalytic kinase), PI3_PI4_kinase (Catalytic kinase), FATC and FAT (Accessory domain for PI34K domains), FHA (Nuclear signaling), HATPase_c (ATPase catalytic activity), C1_1 (Phospholipid binding) and PBD (GTPase interaction). These functional domains have very broad and general functions, thus explaining why they appear in all types of fungi and also in the human kinome. The exception is BCDHK_Adom3; this domain is involved in the regulation of the dehydrogenase complex that breaks down branched-chain amino-acids and it is similar to the HATPase_c family [19]. Interestingly, however, these are, as mentioned, the 10 most common accessory domains in fungi, while in human only two of them, Pkinase_C and C1_1, are also among the 10 most common domains. The remaining six domains that do exist in the human kinome are far less common than in fungi, indicating that these kinase associated domains could be good indicator of functional differences among species.

We believe that the kinome domain distribution, both in type and number, should be indicative of functional and evolutionary differences between fungi. In the next section, we show that this variability is enough to differentiate among different fungal phyla and subphyla.

\section{Domain analysis and Principal Component Analysis grouping}

Taxonomic classification is not always a straightforward task. In this work we tested several criteria for clustering all 30 fungi studied here, based on the number and type of kinases and additional domains (see Methods). Following that, we compared the results with fungal taxonomy $[20,21]$. We found that the most informative clustering was achieved when considering only the distribution of the most common accessory domains. Figure 4 shows the PCA clustering of the different fungi, based on the frequency and type of the 21 most common domains found among all fungal kinomes (see Methods). This classification yields 3 clusters of fungi which show a high correspondence with classical taxonomic classification.

As illustrated in Figure 4, the PCA grouping shows that the domain distribution is more similar among the Pezizomycota subphylum of the Ascomycota and among the Saccharomycota subphylum of the Ascomycota. Among the Basidiomycetes the domain distribution is less tight, although still distinguishable from the first two groups. Though only five species are currently available for analysis, it is noticeable that M. globosa and $U$. maydis both belong to the subphylum Ustilaginomycotina and are fairly close to each other (dots 20 and 22).

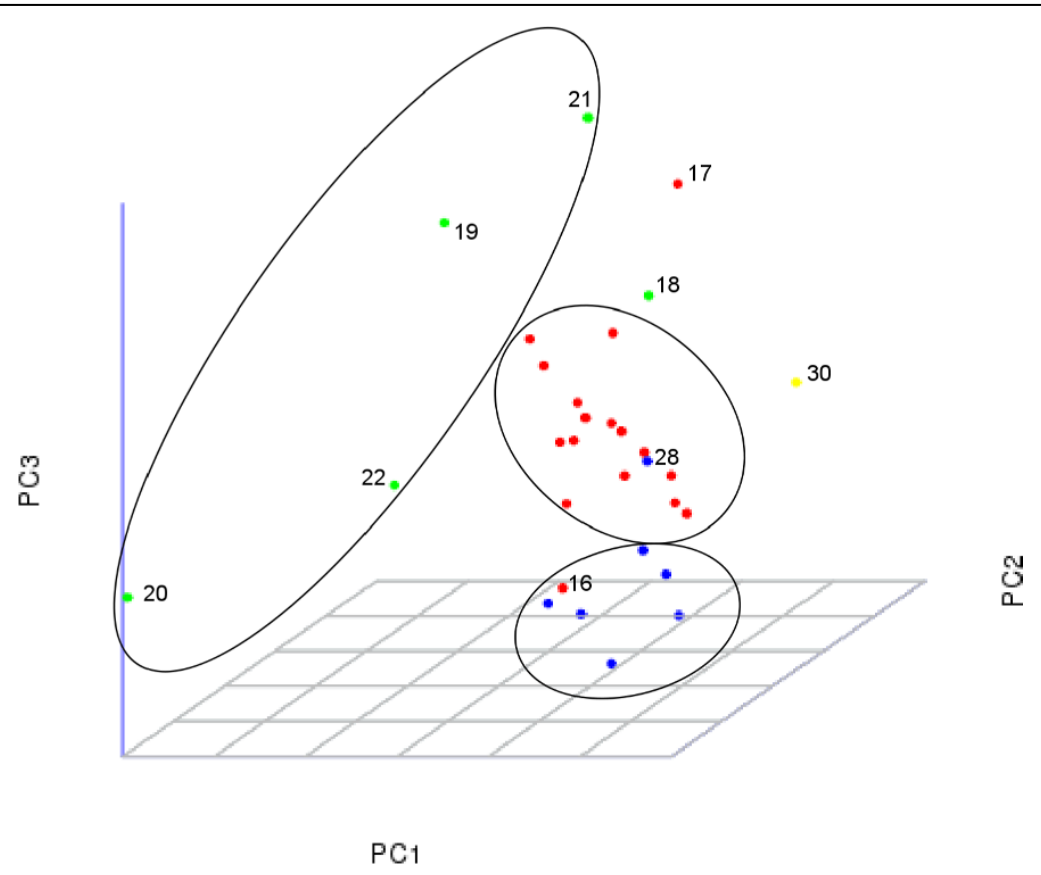

Figure 4 Principal Component Analysis (PCA) of domain frequencies. The PCA plot is based on 21 domain frequencies in all 30 fungi (i.e. domains present in at least half of the fungi, see Additional File 4), projected onto three uncorrelated axes (principal components). The PCA analysis revealed two tight clusters for Ascomycota/Pezizomycota (red dots) and Saccharomycotina (blue dots) groups, and a more spread, but still distinguishable, cluster for Basidiomycetes (green dots). Ellipsoids enclose the clusters for clarity. The only representative of Ascomycota/ Taphrinomycotina (S. pombe) dot 30 (in yellow), forms its own singleton cluster. Species numbers (according to Table 1) are given for some dots, for reference in the text. 
Likewise, dots 18, 19 and 21, which represent members of the Agaricomycotina: $C$. neoformans, $P$. chrysosporium and Laccaria bicolor, are closely located. Assuming that $S$. pombe forms its own singleton cluster, 26 members of the fungi family in this study are clustered by the PCA into one of four groups according to their domain distribution. Four species fall outside these clusters. Of those, two do not reside in any group, and two are mixed: among the ascomycetes, one member of the Pezizomycota (dot 16, Figure 4) and one member of the Saccharomycotina (dot 28, Figure 4) crossed to the other side of the cluster boundaries. Very interestingly, here again $S$. pombe (Ascomycota/Taphrinomycotina), clusters differently from the Pezizomycotina and Saccharomycotina groups. Indeed, when looking closely at the domain content, there are several examples in which $S$. pombe is quite different from its ascomycete relatives. For example, it has a much lower density of HATPase_c domains, a higher percentage of HEAT domains and a higher percentage of the Rapamycin_bind domains. It would be difficult to conclude whether this reflects any aspect of fission yeast lifestyle, or is simply a consequence of evolutionary distance among the species. The PCA results indicate clearly that the domain distribution includes different evolutionary information at the level of the subphyla.

While the classification, based on the most common functional domains from the kinome, is very similar to the classical taxonomy it would also be interesting to try and understand the functional and evolutionary implications of the rare domains appearing only in a certain phyla or fungal species. For example, the ascomycete A. nidulans has unique domains like $\mathrm{CNH}, \mathrm{FAD}$-oxidase_C, RCC1 and many more, making a total of 16 unique accessory domains, which suggests that $A$. nidulans has possibly acquired a diverse kinase-related functionality. Currently, based on its physiology, there is no obvious clue as to why this should be so, but since $A$. nidulans is one of the beststudied model genetic species there may be a good basis to understand this result in future studies.

From the most common domain distribution, there are some clear cases of variations between the subgroups. For example the POLO_box domain appears in all Basidiomycota and in the Saccharomycotina, while is quite rare within the filamentous Ascomycota. Polo boxes appear to mediate interaction with multiple proteins through protein-protein interactions. The HEAT domain, common in both Ascomycete subphyla Pezizomycotina and Saccharomycotina, is extremely rare in Basidiomycetes (appears only in C. neoformans). Many HEAT repeat-containing proteins are involved in intracellular transport processes. Although we cannot fully understand how these differences directly impact the function of the different species, there is a clear correlation between the domain distributions and the taxonomic classification.

\section{Conclusions}

The overall distribution of protein kinases within very different fungal phyla and subphyla seems to be very similar. The overall kinome density is in good agreement with taxonomy. The distribution of additional domains, which could have functional implications, does differ significantly between species, and seems able to provide a functional classification that overlaps with taxonomical classification. Although generally the classical phyla classification correlates with the kinome density and domain distribution, there are exceptions. Basidiomycota do not cluster by kinase number, but they have a similar kinome to proteome ratio. Ascomycetes are well clustered by all criteria, with two exceptions: $A$. nidulans has a different kinome to proteome ratio and a different kinase distribution. Nevertheless, $A$. nidulans is not unusual according to the PCA analysis. Among the filamentous ascomycetes, there is no obvious clustering according to class within the subphylum Pezizomycotina. We note, however, that the class Eurotiomycetes is over-represented in the sequenced genomes published to date, perhaps because the beneficial (Penicillium, Aspergillus oryzae), harmful (Aspergillus nidulans), or pathogenic (Coccidioides, Histoplasma) members of this group, which have drawn much attention over the years. Schizosaccharomyces pombe has a very high kinome density much similar to the Saccharomycotina group. The predicted proteome of Laccaria bicolor has an extraordinary number of TKL kinases; further work can determine whether this is an anomaly, or a more general trait found in mycorrhizal symbionts [22]. Finally, the PCA approach based on the most common domains clusters the Pezizomycotina group and the Saccaromycotina group very tightly, while the Basidiomycetes are more divergent. The approach taken here could be repeated for additional groups of proteins (e.g. G-protein coupled receptors) in order to study their evolution and variability within each fungi phylum. These data can also be used to guide experimental work to elucidate the function of individual protein kinases and the signal transduction pathways they function in.

\section{Methods}

\section{Kinase collection and analysis pipeline}

We have designed and implemented an automatic pipeline (Figure 5) to extract all putative kinases from fungi proteomes and explore their properties. The pipeline uses a variety of tools to extract and classify the putative kinases from all the fungi. Below we describe the pipeline, which can be downloaded as Additional File 2. 


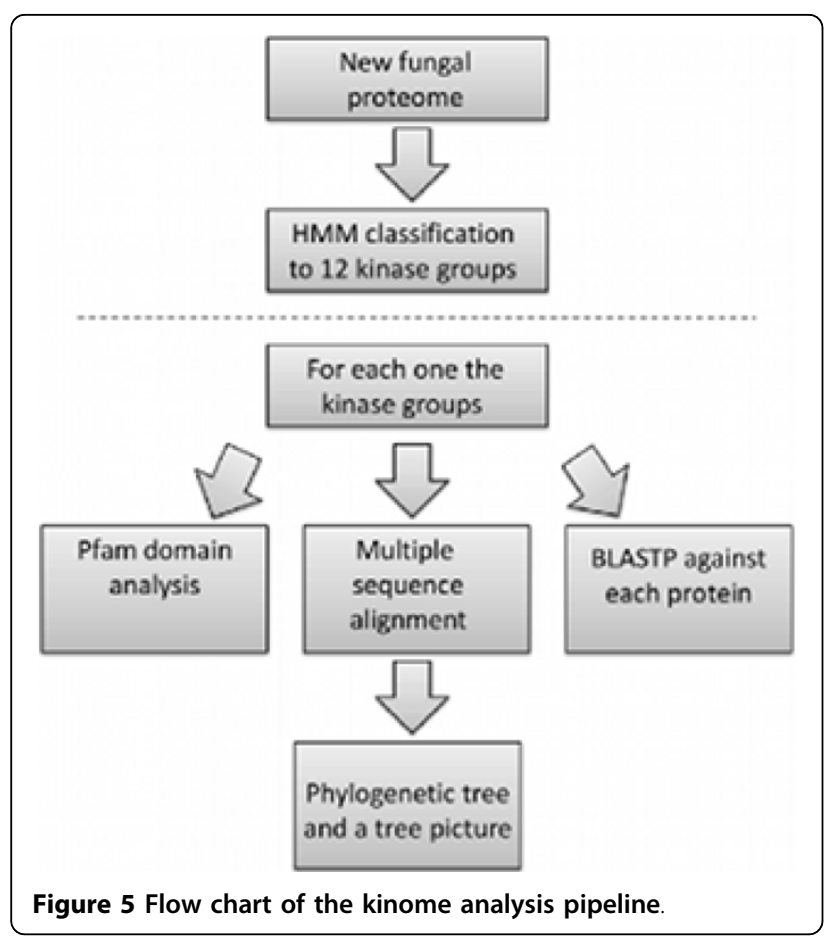

Kinase searching and classification

In order to extract the kinome we fed the HMMer software (version 2.3.2 [23]) with pre-calculated multilevel HMM libraries from the Kinomer 1.0 database. HMMer uses profile hidden Markov models (HMMs) to do sensitive database searching using statistical descriptions of a sequence family's consensus. This process allows an accurate extraction and classification of protein kinases into one of the 12 previously defined kinase groups [8]. Our procedure is very similar to that described by [8], the only significant difference is the cutoff value used for each putative kinase. While Miranda-Saavedra and coworkers [8] used a variable e-value cutoff to choose the correct group for each kinase, we used a fixed HMM bit score cutoff: if the score is greater than 20 a protein is predicted to be a kinase, and if more than one HMM is matched, the higher is selected. Miranda-Saavedra and coworkers reported a high accuracy of their HMM, which according to their study are able to identify successfully between 90 and $97.5 \%$ of all the ePKs of 37 annotated kinomes; from those $\sim 98 \%$ were estimated to be correctly classified in each of the subgroups [8]. Therefore we believe that our results should present a similar accuracy, despite the fact that there might be a few mis-classifications in any group of kinases. The modified cutoff criteria used here (see Methods) resulted in slightly different numbers of predicted kinases when compared to the Kinomer results, but the calculated distribution (data not shown) is very similar, for those species previously studied [8].
We chose a fixed bit score cutoff, since it provides us with a unified criterion for all fungi, so we can compare the numbers based on the same scale. The bit score reflects whether the sequence is a good match to the HMM model. A score above $\log _{2}$ of the number of sequences in the target database is likely to be a true homologue. For our fungi proteomes, this rule-of-thumb number is on the order of 20 bits. In any case e-value and bit score should be strongly correlated, true homologues will have both a good bit score and a good Evalue [23].

Once the kinase groups are populated, the pipeline is designed to extract functional and phylogenetic information from the list of putative kinases. The pipeline procedure and subsequent analysis (see below) is summarized in Figure 5. Since we preferred here to miss a number of potential kinases rather than including false positives, we did not use the category "Others" as described in the Kinomer database.

\section{Functional information}

Homology search - The pipeline runs Blastp [24] (version 2.2.17) against the Swissprot database (November 2008 version) to allow comparison to known proteins with annotation. The output of this stage is a list of all homologs for each kinase entry. Data from this stage is not shown in the paper.

Domains search - The pipeline identifies the domains of each putative kinase by running the pfam_search.pl script against PFAM A [25] HMMs library. We then analyze the presence of each domain within a specific fungal group.

\section{Phylogenetic information}

In order to build phylogenetic trees we constructed multiple alignments for each group using the MSA program MUSCLE (version 3.7) [26,27]. The multiple sequence alignments are then used for constructing phylogenetic trees using FastTree version 2.0 [28] with the generalized time-reversible models of nucleotide evolution and the JTT model [29] of amino acid evolution. We used FigTree http://tree.bio.ed.ac.uk/software/figtree/ for phylogenetic visualization. Additional File 3 shows one example of the phylogenetic trees produced. This corresponds to the 134 predicted kinases of Aspergillus nidulans. Aside from a few exceptions, the kinases are clustered according to their predicted group.

The pipeline is written in Perl scripting language and was tested on Fedora and Ubuntu operating systems.

\section{Principal Component Analysis (PCA)}

We classified the 30 fungi based on the percentage of each domain type found in each species, limiting the data to those domains present in at least half of the 
fungi (see Additional File 4). We then applied the PCA procedure described in [30] to cluster the fungi. The PCA was obtained using the GNU R software (R: A Language and Environment for Statistical Computing, http://www.R-project.org, 2009).

\section{Significance test}

The Wilcoxon-Mann-Whitney test was performed on the kinome density values of our three phylogenic groups using GNU R software (R: A Language and Environment for Statistical Computing, http://www.rproject.org/index.html) and the wilcox.test function with its non paired mode.

\section{Additional File 1: Total predicted number of kinases per fungal}

species. Bar colors indicate significantly different kinome size (see text for details).

Click here for file

[http://www.biomedcentral.com/content/supplementary/1471-2164-11133-S1.EPS ]

Additional File 2: Pipeline for kinome analysis. This file contains the pipeline software and documentation.

Click here for file

[http://www.biomedcentral.com/content/supplementary/1471-2164-11133-S2.RAR ]

Additional File 3: Phylogenetic tree of the Aspergillus nidulans kinome. Taxa names are composed of the predicted kinase group followed by an underscore and the protein code as it appears in the original proteome. Simulated bootstrapped values are also shown.

Click here for file

[http://www.biomedcentral.com/content/supplementary/1471-2164-11133-S3.EPS ]

Additional File 4: Domain percentage data used as input for the PCA analysis. The first column lists the species names, abbreviated according to Table 1. The values are the percentages of the domains indicated in each column, according to PFAM notation.

Click here for file

[http://www.biomedcentral.com/content/supplementary/1471-2164-11133-S4.PNG ]

\section{Acknowledgements}

IK was supported by a fellowship from the Technion Graduate School, and in part by the US-Israel Binational Agricultural Research and Development Fund (TIE-BARD 8031-08). We are grateful to Jeffrey Rollins and Martin B. Dickman for their assent to our analysis of $S$. sclerotiorum prior to publication of the genome paper.

\section{Author details}

${ }^{1}$ Department of Biology, Technion - Israel Institute of Technology, Haifa 32000, Israel. ${ }^{2}$ Bioinformatics Knowledge Unit, The Lorry I. Lokey Interdisciplinary Center for Life Sciences and Engineering, Technion - Israel Institute of Technology, Haifa 32000, Israel.

\section{Authors' contributions}

IK participated in the design of the study, set up and tested the computational tools and did the major part of the analysis; YMG participated in the design and coordination of the project and in data interpretation; $\mathrm{BAH}$ initiated our interest in fungal protein kinases and provided the biological context; FG conceived of the bioinformatics study, designed it, and did the domain analysis. FG and BAH drafted the manuscript; IK and YMG helped draft the manuscript. All authors read and approved the final manuscript.

\section{Authors' information}

IK is a graduate student in the structural biology and bioinformatics lab led by YMG at the Department of Biology, Technion - Israel Institute of Technology. BAH, also at the Department of Biology, Technion, leads a lab studying signal transduction pathways of filamentous fungi, and is currently involved in annotation for several fungal genome projects. FG is a staff member of the Bioinformatics Knowledge Unit at the Technion - Israel Institute of Technology.

Received: 25 August 2009

Accepted: 24 February 2010 Published: 24 February 2010

\section{References}

1. Bahn YS, Xue C, Idnurm A, Rutherford JC, Heitman J, Cardenas ME: Sensing the environment: lessons from fungi. Nat Rev Microbiol 2007, 5(1):57-69.

2. Wood V, Gwilliam R, Rajandream MA, Lyne M, Lyne R, Stewart A, Sgouros J, Peat N, Hayles J, Baker S, et al: The genome sequence of Schizosaccharomyces pombe. Nature 2002, 415(6874):871-880.

3. Hemmings BA, Restuccia D, Tonks N: Targeting the Kinome II. Curr Opin Cell Biol 2009, 21(2):135-139.

4. Caenepeel S, Charydczak G, Sudarsanam S, Hunter T, Manning G: The mouse kinome: discovery and comparative genomics of all mouse protein kinases. Proc Natl Acad Sci USA 2004, 101(32):11707-11712.

5. Manning G, Plowman GD, Hunter T, Sudarsanam S: Evolution of protein kinase signaling from yeast to man. Trends Biochem Sci 2002, 27(10):514-520.

6. Manning G, Whyte DB, Martinez R, Hunter T, Sudarsanam S: The protein kinase complement of the human genome. Science 2002, 298(5600):1912-1934.

7. Hanks SK, Hunter T: Protein kinases 6. The eukaryotic protein kinase superfamily: kinase (catalytic) domain structure and classification. Faseb $J$ 1995, 9(8):576-596.

8. Miranda-Saavedra D, Barton GJ: Classification and functional annotation of eukaryotic protein kinases. Proteins 2007, 68(4):893-914.

9. $\mathrm{Xu} J \mathrm{R}$, Peng $\mathrm{YL}$, Dickman MB, Sharon $\mathrm{A}$ : The dawn of fungal pathogen genomics. Annu Rev Phytopathol 2006, 44:337-366.

10. Martin DM, Miranda-Saavedra D, Barton GJ: Kinomer v. 1.0: a database of systematically classified eukaryotic protein kinases. Nucleic Acids Res 2009, 37 Database: D244-250.

11. Cornell MJ, Alam I, Soanes DM, Wong HM, Hedeler C, Paton NW, Rattray M, Hubbard SJ, Talbot NJ, Oliver SG: Comparative genome analysis across a kingdom of eukaryotic organisms: specialization and diversification in the fungi. Genome Res 2007, 17(12):1809-1822.

12. Maere S, De Bodt S, Raes J, Casneuf T, Van Montagu M, Kuiper M, Peer Van de $Y$ : Modeling gene and genome duplications in eukaryotes. Proc Natl Acad Sci USA 2005, 102(15):5454-5459.

13. Thomas BC, Pedersen B, Freeling M: Following tetraploidy in an Arabidopsis ancestor, genes were removed preferentially from one homeolog leaving clusters enriched in dose-sensitive genes. Genome Res 2006, 16(7):934-946.

14. Ma $\sqcup$, Ibrahim AS, Skory C, Grabherr MG, Burger G, Butler M, Elias M, Idnurm A, Lang BF, Sone T, et al: Genomic analysis of the basal lineage fungus Rhizopus oryzae reveals a whole-genome duplication. PLoS Genet 2009, 5(7):e1000549.

15. Perez J, Castaneda-Garcia A, Jenke-Kodama H, Muller R, Munoz-Dorado J: Eukaryotic-like protein kinases in the prokaryotes and the myxobacterial kinome. Proc Natl Acad Sci USA 2008, 105(41):15950-15955.

16. Colot HV, Loros JJ, Dunlap JC: Temperature-modulated alternative splicing and promoter use in the Circadian clock gene frequency. Mol Biol Cell 2005, 16(12):5563-5571.

17. Diernfellner A, Colot HV, Dintsis O, Loros JJ, Dunlap JC, Brunner M: Long and short isoforms of Neurospora clock protein FRQ support temperature-compensated circadian rhythms. FEBS Lett 2007, 581(30):5759-5764

18. James TY, Kauff F, Schoch CL, Matheny PB, Hofstetter V, Cox CJ, Celio G, Gueidan C, Fraker E, Miadlikowska J, et al: Reconstructing the early evolution of Fungi using a six-gene phylogeny. Nature 2006, 443(7113):818-822.

19. Popov KM, Zhao Y, Shimomura Y, Kuntz MJ, Harris RA: Branched-chain alpha-ketoacid dehydrogenase kinase. Molecular cloning, expression, 
and sequence similarity with histidine protein kinases. J Biol Chem 1992, 267(19):13127-13130

20. Maddison DR, Schulz K-S, Maddison WP: The Tree of Life Web Project. Linnaeus Tercentenary: Progress in Invertebrate Taxonomy Zhang Z-QS, W A 2007, 1668:1-766.

21. Hibbett DS, Binder M, Bischoff JF, Blackwell M, Cannon PF, Eriksson OE, Huhndorf S, James T, Kirk PM, Lucking R, et al: A higher-level phylogenetic classification of the Fungi. Mycol Res 2007, 111(Pt 5):509-547.

22. Rajashekar B, Kohler A, Johansson T, Martin F, Tunlid A, Ahren D: Expansion of signal pathways in the ectomycorrhizal fungus Laccaria bicolorevolution of nucleotide sequences and expression patterns in families of protein kinases and RAS small GTPases. New Phytol 2009, 183(2):365-379.

23. Eddy SR: Profile hidden Markov models. Bioinformatics 1998, 14(9):755-763.

24. Altschul SF, Gish W, Miller W, Myers EW, Lipman DJ: Basic local alignment search tool. J Mol Biol 1990, 215(3):403-410.

25. Finn RD, Tate J, Mistry J, Coggill PC, Sammut SJ, Hotz HR, Ceric G, Forslund K, Eddy SR, Sonnhammer EL, et al: The Pfam protein families database. Nucleic Acids Res 2008, 36 Database: D281-288.

26. Edgar RC: MUSCLE: multiple sequence alignment with high accuracy and high throughput. Nucleic Acids Res 2004, 32(5):1792-1797.

27. Edgar RC: MUSCLE: a multiple sequence alignment method with reduced time and space complexity. BMC Bioinformatics 2004, 5:113.

28. Nierman WC, Pain A, Anderson MJ, Wortman JR, Kim HS, Arroyo J, Berriman M, Abe K, Archer DB, Bermejo C, et al: Genomic sequence of the pathogenic and allergenic filamentous fungus Aspergillus fumigatus. Nature 2005, 438(7071):1151-1156.

29. Jones $D$, Taylor $W$, Thornton J: The rapid generation of mutation data matrices from protein sequences. Comput Appl Biosci 1992, 8(3):275-282.

30. Tzahor S, Man-Aharonovich D, Kirkup BC, Yogev T, Berman-Frank I, Polz MF, Beja O, Mandel-Gutfreund Y: A supervised learning approach for taxonomic classification of core-photosystem-II genes and transcripts in the marine environment. BMC Genomics 2009, 10:229.

31. Qin X, Evans JD, Aronstein KA, Murray KD, Weinstock GM: Genome sequences of the honey bee pathogens Paenibacillus larvae and Ascosphaera apis. Insect Mol Biol 2006, 15(5):715-718.

32. Fedorova ND, Khaldi N, Joardar VS, Maiti R, Amedeo P, Anderson MJ, Crabtree J, Silva JC, Badger JH, Albarraq A, et al: Genomic islands in the pathogenic filamentous fungus Aspergillus fumigatus. PLoS Genet 2008, 4(4):e1000046.

33. Galagan JE, Calvo SE, Cuomo C, Ma LJ, Wortman JR, Batzoglou S, Lee SI, Basturkmen M, Spevak CC, Clutterbuck J, et al: Sequencing of Aspergillus nidulans and comparative analysis with $A$. fumigatus and $A$. oryzae. Nature 2005, 438(7071):1105-1115.

34. Pel HJ, de Winde JH, Archer DB, Dyer PS, Hofmann G, Schaap PJ, Turner G, de Vries RP, Albang R, Albermann K, et al: Genome sequencing and analysis of the versatile cell factory Aspergillus niger CBS 513.88. Nat Biotechnol 2007, 25(2):221-231.

35. Sharpton TJ, Stajich JE, Rounsley SD, Gardner MJ, Wortman JR, Jordar VS, Maiti R, Kodira CD, Neafsey DE, Zeng Q, et al: Comparative genomic analyses of the human fungal pathogens Coccidioides and their relatives. Genome Res 2009, 19(10):1722-1731.

36. Cuomo CA, Guldener U, Xu JR, Trail F, Turgeon BG, Di Pietro A, Walton JD, Ma $L$, Baker SE, Rep M, et al: The Fusarium graminearum genome reveals a link between localized polymorphism and pathogen specialization. Science 2007, 317(5843): 1400-1402.

37. Dean RA, Talbot NJ, Ebbole DJ, Farman ML, Mitchell TK, Orbach MJ, Thon M, Kulkarni R, Xu JR, Pan $H$, et al: The genome sequence of the rice blast fungus Magnaporthe grisea. Nature 2005, 434(7036):980-986.

38. Galagan JE, Calvo SE, Borkovich KA, Selker EU, Read ND, Jaffe D, FitzHugh W, Ma L, Smirnov S, Purcell S, et al: The genome sequence of the filamentous fungus Neurospora crassa. Nature 2003, 422(6934):859-868.

39. Berg van den MA, Albang R, Albermann K, Badger JH, Daran JM, Driessen AJ, Garcia-Estrada C, Fedorova ND, Harris DM, Heijne WH, et al: Genome sequencing and analysis of the filamentous fungus Penicillium chrysogenum. Nat Biotechnol 2008, 26(10):1161-1168.

40. Hane JK, Lowe RG, Solomon PS, Tan KC, Schoch CL, Spatafora JW, Crous PW, Kodira C, Birren BW, Galagan JE, et al: Dothideomycete plant interactions illuminated by genome sequencing and EST analysis of the wheat pathogen Stagonospora nodorum. Plant Cell 2007, 19(11):3347-3368.
41. Martinez D, Berka RM, Henrissat B, Saloheimo M, Arvas M, Baker SE, Chapman J, Chertkov O, Coutinho PM, Cullen D, et al: Genome sequencing and analysis of the biomass-degrading fungus Trichoderma reesei (syn. Hypocrea jecorina). Nat Biotechnol 2008, 26(5):553-560.

42. Loftus BJ, Fung E, Roncaglia P, Rowley D, Amedeo P, Bruno D, Vamathevan J, Miranda M, Anderson IJ, Fraser JA, et al: The genome of the basidiomycetous yeast and human pathogen Cryptococcus neoformans. Science 2005, 307(5713):1321-1324.

43. Martin F, Aerts A, Ahren D, Brun A, Danchin EG, Duchaussoy F, Gibon J, Kohler A, Lindquist E, Pereda $V$, et al: The genome of Laccaria bicolor provides insights into mycorrhizal symbiosis. Nature 2008 , 452(7183):88-92

44. Xu J, Saunders CW, Hu P, Grant RA, Boekhout T, Kuramae EE, Kronstad JW, Deangelis YM, Reeder NL, Johnstone KR, et al: Dandruff-associated Malassezia genomes reveal convergent and divergent virulence traits shared with plant and human fungal pathogens. Proc Natl Acad Sci USA 2007, 104(47):18730-18735.

45. Martinez D, Larrondo LF, Putnam N, Gelpke MD, Huang K, Chapman J, Helfenbein KG, Ramaiya P, Detter JC, Larimer F, et al: Genome sequence of the lignocellulose degrading fungus Phanerochaete chrysosporium strain RP78. Nat Biotechnol 2004, 22(6):695-700.

46. Kamper J, Kahmann R, Bolker M, Ma LJ, Brefort T, Saville BJ, Banuett F, Kronstad JW, Gold SE, Muller O, et al: Insights from the genome of the biotrophic fungal plant pathogen Ustilago maydis. Nature 2006, 444(7115):97-101.

47. Dietrich FS, Voegeli S, Brachat S, Lerch A, Gates K, Steiner S, Mohr C, Pohlmann R, Luedi P, Choi $S$, et al: The Ashbya gossypii genome as a tool for mapping the ancient Saccharomyces cerevisiae genome. Science 2004, 304(5668):304-307.

48. Jones T, Federspiel NA, Chibana H, Dungan J, Kalman S, Magee BB, Newport G, Thorstenson YR, Agabian N, Magee PT, et al: The diploid genome sequence of Candida albicans. Proc Natl Acad Sci USA 2004, 101(19):7329-7334.

49. Dujon B, Sherman D, Fischer G, Durrens P, Casaregola S, Lafontaine I, De Montigny J, Marck C, Neuveglise C, Talla E, et al: Genome evolution in yeasts. Nature 2004, 430(6995):35-44

50. Jeffries TW, Grigoriev IV, Grimwood J, Laplaza JM, Aerts A, Salamov A, Schmutz J, Lindquist E, Dehal P, Shapiro H, et al: Genome sequence of the lignocellulose-bioconverting and xylose-fermenting yeast Pichia stipitis. Nat Biotechnol 2007, 25(3):319-326.

51. Goffeau A, Barrell BG, Bussey H, Davis RW, Dujon B, Feldmann H, Galibert F, Hoheisel JD, Jacq C, Johnston M, et al: Life with 6000 genes. Science 1996, 274(5287):546

doi:10.1186/1471-2164-11-133

Cite this article as: Kosti et al:: Comparative analysis of fungal protein kinases and associated domains. BMC Genomics 2010 11:133.

\section{Submit your next manuscript to BioMed Central and take full advantage of:}

- Convenient online submission

- Thorough peer review

- No space constraints or color figure charges

- Immediate publication on acceptance

- Inclusion in PubMed, CAS, Scopus and Google Scholar

- Research which is freely available for redistribution 